Journal of Applied AnALysis

Vol. 6, No. 2 (2000), pp. 251-258

\title{
QUOTIENTS OF QUASI-CONTINUOUS FUNCTIONS
}

\author{
J. JAEOCHA \\ Received March 23, 2000 and, in revised form, June 15, 2000
}

\begin{abstract}
The main goal of this paper is to characterize both the quotients of quasi-continuous and the quotients of Darboux quasi-continuous functions. We prove also theorems concerning common divisor for the families of the quotients of quasi-continuous (Darboux quasi-continuous) functions with respect to quasi-continuity (Darboux property and quasicontinuity, respectively).
\end{abstract}

\section{Introduction}

The letters $\mathbb{R}, \mathbb{Q}, \mathbb{Z}$, and $\mathbb{N}$ denote the real line, the set of rationals, the set of integers, and the set of positive integers, respectively. The word function denotes a mapping from $\mathbb{R}$ to $\mathbb{R}$ unless otherwise explicitly stated. For each set $A$ we use the symbols int $A, \operatorname{cl} A, \operatorname{bd} A, \chi_{A}$, and card $A$ to denote the interior, the closure, the boundary, the characteristic function, and the cardinality of $A$, respectively. We say that a set $A \subset \mathbb{R}$ is semi-open [8], if $A \subset \operatorname{clint} A$.

Let $f: \mathbb{R} \rightarrow \mathbb{R}$. The symbol $C(f)$ denotes the set of points of continuity of $f$. For each $y \in \mathbb{R}$ let $[f<y]=\{x \in \mathbb{R}: f(x)<y\}$. Similarly we define

1991 Mathematics Subject Classification. Primary 26A15; Secondary 54C08.

Key words and phrases. Darboux function, quasi-continuous function, quotient of functions.

ISSN 1425-6908 CHeldermann Verlag. 
the symbols $[f>y],[f=y]$, etc. If $A \subset \mathbb{R}$ is nonempty, then let $\operatorname{osc}(f, A)$ be the oscillation of $f$ on $A$, i.e., $\operatorname{osc}(f, A)=\sup \{|f(x)-f(t)|: x, t \in A\}$.

The following denote classes of functions:

$\mathcal{D}$ : consists of all Darboux functions, i.e., $f \in \mathcal{D}$ iff it has the intermediate value property;

$\mathcal{Q}$ : consists of all quasi-continuous functions in the sense of Kempisty [7]; recall that $f \in \mathcal{Q}$ iff for each $x \in \mathbb{R}$ there is a sequence $\left(x_{n}\right) \subset C(f)$ such that $x_{n} \rightarrow x$ and $f\left(x_{n}\right) \rightarrow f(x)$ (see, e.g., [5] or [6, Lemma 2]);

$\mathcal{D Q}$ : denotes the family of Darboux quasi-continuous functions;

$\mathcal{S}_{s}$ : consists of all strong Światkowski functions [9], i.e., $f \in \mathcal{S}_{s}$ iff for all $a<b$ and $y$ between $f(a)$ and $f(b)$, there is an $x \in(a, b) \cap C(f)$ with $f(x)=y$; one can easily see that $\mathcal{S}_{s} \subset \mathcal{D} \mathcal{Q}$;

$\mathcal{C}_{q}$ : consists of all cliquish functions [15]; recall that $f \in \mathcal{C}_{q}$ iff $\operatorname{cl} C(f)=\mathbb{R}$ (see, e.g., [14]).

There are several papers concerning theorems on a common summand [4], [3], or factor [12]. In this paper we deal with theorems on a common divisor. In particular we characterize the cardinal

$\mathrm{q}(\mathcal{A}) \stackrel{\text { df }}{=} \min \left(\left\{\operatorname{card} \mathcal{F}: \mathcal{F} \subset \mathcal{A} / \mathcal{A} \& \neg\left(\exists_{g} \forall_{f \in \mathcal{F}} f / g \in \mathcal{A}\right)\right\} \cup\left\{(\operatorname{card} \mathcal{A} / \mathcal{A})^{+}\right\}\right)$

for the families $\mathcal{Q}$ and $\mathcal{D} \mathcal{Q}$, where

$$
\mathcal{A} / \mathcal{A} \stackrel{\mathrm{df}}{=}\{f / g: f, g \in \mathcal{A} \& g(x) \neq 0 \text { for each } x \in \mathbb{R}\} .
$$

In the above definition it is quite natural to restrict ourselves to subfamilies of $\mathcal{A} / \mathcal{A}$ only. Indeed, if there is a function $g$ such that both $f / g$ and $1 / g$ are in $\mathcal{A}$, then $f \in \mathcal{A} / \mathcal{A}$. Therefore, before we can examine the value of $\mathrm{q}(\mathcal{A})$, we should know what the family $\mathcal{A} / \mathcal{A}$ is.

\section{Quasi-continuous functions}

Denote by $\mathcal{B}$ the family of all cliquish functions $f$ such that the set $[f \neq 0]$ is semi-open. We start with a simple proposition.

Proposition 2.1. $\mathcal{Q} / \mathcal{Q} \subset \mathcal{B}$.

Proof. Let $f=g / h$, where $g, h \in \mathcal{Q}$. Then the cliquishness of $f$ is obvious. By $[14]$, the set $[g \neq 0]$ is semi-open. Clearly $[f \neq 0]=[g \neq 0]$.

Our next goal is to show that $\mathcal{Q} / \mathcal{Q}=\mathcal{B}$. 
Lemma 2.2. Let $I=[a, b]$ and $m<M$. Suppose that $f_{1}, \ldots, f_{l} \in \mathcal{C}_{q}$, and $\max \left\{\operatorname{osc}\left(f_{i}, I\right): i \in\{1, \ldots, l\}\right\}<1$. There is a Baire one function $g$ such that $g=0$ on $\operatorname{bd} I$, and for each $i,\left(f_{i}+g\right)[I] \supset[m, M]$ and $\left(f_{i}+g\right)\lceil I$ is strong Światkowski.

Proof. Put

$$
\begin{aligned}
& \widetilde{m}=m-\max \left\{\sup \left|f_{i}\right|[I]: i \in\{1, \ldots, l\}\right\}-1, \\
& \widetilde{M}=M+\max \left\{\sup \left|f_{i}\right|[I]: i \in\{1, \ldots, l\}\right\}+1 .
\end{aligned}
$$

Let $\varphi$ be a continuous function such that $\varphi[I] \supset[\widetilde{m}, \widetilde{M}]$ and $\varphi=0$ on bd $I$. For each $i$ define $\tilde{f}_{i}=\left(f_{i}+\varphi\right) \chi_{I}+f_{i}(a) \chi_{(-\infty, a)}+f_{i}(b) \chi_{(b, \infty)}$. By [10, Theorem 4], there is a Baire one function $\tilde{g}$ such that $\widetilde{f}_{i}+\tilde{g} \in \mathcal{S}_{s}$ for each $i$, and $\sup |\tilde{g}|[\mathbb{R}]<1$; by its proof, we can conclude that $\tilde{g}=0$ on bd $I$.

Put $g=\varphi+\tilde{g}$. Then for each $i,\left(f_{i}+g\right) \mid I$ is strong Świątkowski and

$$
\begin{aligned}
\left(f_{i}+g\right)[I] & \supset\left(\inf \left(f_{i}+g\right)[I], \sup \left(f_{i}+g\right)[I]\right) \\
& \supset\left[\sup f_{i}[I]+\widetilde{m}+1, \inf f_{i}[I]+\widetilde{M}-1\right] \supset[m, M] .
\end{aligned}
$$

Clearly $g$ is Baire one and $g=0$ on bd $I$.

Lemma 2.3. Let $f_{1}, \ldots, f_{l} \in \mathcal{C}_{q}$, and assume that each $f_{i}$ is either positive or negative on $(a, b)$. There is a Baire one function $g:(a, b) \rightarrow \mathbb{R} \backslash\{0\}$ such that for each $i,\left(f_{i} / g\right)[(a, c)]=\left(f_{i} / g\right)[(c, b)]=\mathbb{R} \backslash\{0\}$ for each $c \in(a, b)$, and $f_{i} / g$ is quasi-continuous.

Proof. For each $i$ define

$$
\tilde{f}_{i}(x)= \begin{cases}\ln \left|f_{i}\right|(x) & \text { if } x \in(a, b), \\ 0 & \text { otherwise. }\end{cases}
$$

Clearly each $\tilde{f}_{i}$ is cliquish. So by [10, Theorem 4], there is a Baire one function $\bar{g}$ such that $\tilde{f}_{i}-\bar{g} \in \mathcal{S}_{s}$ for each $i$. Let $\left\{a_{z}: z \in \mathbb{Z}\right\} \subset \bigcap_{i=1}^{l} C\left(\widetilde{f}_{i}-\bar{g}\right)$ be an increasing sequence with limit points $a$ and $b$. Fix $z \in \mathbb{Z}$. Choose $b_{z} \in\left(a_{z}, a_{z+1}\right)$ such that $\max \left\{\operatorname{osc}\left(\widetilde{f}_{i}-\bar{g},\left[a_{z}, b_{z}\right]\right): i \in\{1, \ldots, l\}\right\}<1$. Use Lemma 2.2 to construct a Baire one function $g_{z}$ such that $g_{z}=0$ on $\left\{a_{z}, b_{z}\right\}$, and for each $i,\left(\widetilde{f}_{i}-\bar{g}-g_{z}\right) \uparrow\left[a_{z}, b_{z}\right]$ is strong Świątkowski and $\left(\widetilde{f}_{i}-\bar{g}-g_{z}\right)\left[\left[a_{z}, b_{z}\right]\right] \supset[-|z|,|z|]$. Define

$$
\tilde{g}(x)= \begin{cases}\bar{g}(x)+g_{z}(x) & \text { if } x \in\left[a_{z}, b_{z}\right], z \in \mathbb{Z}, \\ \bar{g}(x) & \text { otherwise, }\end{cases}
$$

and let $g(x)=(-1)^{z} \exp (\tilde{g}(x))$ if $x \in\left[a_{z}, a_{z+1}\right), z \in \mathbb{Z}$. We will prove that $g$ has all required properties. Clearly $g$ is Baire one. 
Fix $i \in\{1, \ldots, l\}$. Notice that for each $z, f_{i} / g=(-1)^{z} \exp \circ\left(\widetilde{f}_{i}-\right.$ $\tilde{g}) \operatorname{sgn} \circ f_{i}$ on $\left[a_{z}, a_{z+1}\right)$ and $\left(\widetilde{f}_{i}-\tilde{g}\right) \uparrow\left[a_{z}, a_{z+1}\right)$ is quasi-continuous. It follows that $f_{i} / g$ is quasi-continuous on $(a, b)=\bigcup_{z \in \mathbb{Z}}\left[a_{z}, a_{z+1}\right)$.

Finally let $c \in(a, b)$ and $y \neq 0$. Choose a $z \in \mathbb{Z}$ with $|\ln | y|| \leq|z|$ such that $\left[a_{z}, b_{z}\right] \subset(a, c)$ and $(-1)^{z}=\operatorname{sgn} y \cdot \operatorname{sgn} \circ f_{i}$ on $(a, b)$. Since $\left(\widetilde{f}_{i}-\right.$ $\tilde{g})\left[\left[a_{z}, b_{z}\right]\right] \supset[-|z|,|z|]$, there is an $x \in\left[a_{z}, b_{z}\right]$ such that $\ln |y|=\ln \left|f_{i}\right|(x)-$ $\tilde{g}(x)$. Thus

$$
y=|y| \operatorname{sgn} y=\left(\left|f_{i}\right| / \exp \circ \widetilde{g}\right)(x)(-1)^{z} \operatorname{sgn} f_{i}(x)=\left(f_{i} / g\right)(x) .
$$

Similarly we can show that $y=\left(f_{i} / g\right)\left(x^{\prime}\right)$ for some $x^{\prime} \in(c, b)$.

Remark. We say that a set $A \subset \mathbb{R}$ is simply open [1] if bd $A$ is nowhere dense. It is easy to show that each semi-open set is simply open. So by [2], if $f \in \mathcal{B}$, then the set int $[f=0] \cup \operatorname{int}[f>0] \cup \operatorname{int}[f<0]$ is dense in $\mathbb{R}$.

Theorem 2.4. Let $f_{1}, \ldots f_{k} \in \mathcal{B}$. There is a Baire one function $g: \mathbb{R} \rightarrow$ $\mathbb{R} \backslash\{0\}$ such that $f_{i} / g \in \mathcal{Q}$ for each $i$.

Proof. Let $\left\{\left(a_{n}, b_{n}\right): n<r\right\}$, where $r \leq \omega$, be a sequence of all components of the set

$$
U=\bigcap_{i=1}^{k}\left(\operatorname{int}\left[f_{i}=0\right] \cup \operatorname{int}\left[f_{i}>0\right] \cup \operatorname{int}\left[f_{i}<0\right]\right) .
$$

By the remark preceding this theorem, $U$ is dense in $\mathbb{R}$. Clearly sgn $\circ f_{i}$ is constant on $\left(a_{n}, b_{n}\right)$ for each $i$ and $n$. By Lemma 2.3, for each $n$ there is a Baire one function $g_{n}:\left(a_{n}, b_{n}\right) \rightarrow \mathbb{R} \backslash\{0\}$ such that for each $i, f_{i} / g_{n}$ is quasi-continuous on $\left(a_{n}, b_{n}\right)$ and if $f_{i}$ is nonzero on $\left(a_{n}, b_{n}\right)$, then

$$
\begin{aligned}
& \left(f_{i} / g_{n}\right)\left[\left(a_{n}, c\right)\right] \\
& =\left(f_{i} / g_{n}\right)\left[\left(c, b_{n}\right)\right]=\mathbb{R} \backslash\{0\} \quad \text { for each } c \in\left(a_{n}, b_{n}\right) .
\end{aligned}
$$

Define

$$
g(x)= \begin{cases}g_{n}(x) & \text { if } x \in\left(a_{n}, b_{n}\right), n<r \\ 1 & \text { otherwise }\end{cases}
$$

Fix $i \in\{1, \ldots, k\}$. Clearly $f_{i} / g$ is quasi-continuous on $U$. So, let $x \in$ $\mathbb{R} \backslash U$. We consider two cases.

If $x \notin \operatorname{cl} \operatorname{int}\left[f_{i} \neq 0\right]$, then since $\left[f_{i} \neq 0\right]$ is semi-open, $x \notin \operatorname{cl}\left[f_{i} \neq 0\right]$. Hence $x \in \operatorname{int}\left[f_{i}=0\right]=\operatorname{int}\left[\left(f_{i} / g\right)=0\right]$, and $f_{i} / g$ is continuous at $x$.

In the opposite case, there is a sequence $\left(I_{m}\right) \subset\left\{\left(a_{n}, b_{n}\right) \cap\left[f_{i} \neq 0\right]\right.$ : $n<r\}$ such that $\varrho\left(x, I_{m}\right) \rightarrow 0$, where $\varrho\left(x, I_{m}\right) \stackrel{\text { df }}{=} \inf \left\{|x-t|: t \in I_{m}\right\}$. (We use the fact that $U$ is dense in $\mathbb{R}$, and the definition of $\mathcal{B}$.) Notice 
that $f_{i} / g$ is quasi-continuous on each $I_{m}$. So by (2), there is a sequence $\left(x_{m}\right) \subset C\left(f_{i} / g\right)$ such that $x_{m} \rightarrow x$ and $\left(f_{i} / g\right)\left(x_{m}\right) \rightarrow\left(f_{i} / g\right)(x)$. So, $f_{i} / g$ is quasi-continuous at $x$.

Corollary 2.5. $\mathcal{Q} / \mathcal{Q}=\mathcal{B}$.

Proof. Let $f \in \mathcal{B}$. By Theorem 2.4, there is a function $g$ such that $f / g \in \mathcal{Q}$ and $1 / g \in \mathcal{Q}$. Hence $f=(f / g) /(1 / g) \in \mathcal{Q} / \mathcal{Q}$.

The opposite inclusion follows by Proposition 2.1.

Proposition 2.6. For each function $g: \mathbb{R} \rightarrow \mathbb{R} \backslash\{0\}$ there is a $q \in \mathbb{Q}$ such that $\left(\exp \circ \chi_{\{q\}}\right) / g \notin \mathcal{Q}$.

Proof. If $C(g)=\emptyset$, then $C\left(\left(\exp \circ \chi_{\{0\}}\right) / g\right) \subset\{0\}$, so $\left(\exp \circ \chi_{\{0\}}\right) / g \notin \mathcal{Q}$. Hence

Otherwise by [11, Proposition 3.3], $\chi_{\{q\}}-\ln |g| \notin \mathcal{Q}$ for some $q \in \mathbb{Q}$.

$$
\exp \circ\left(\chi_{\{q\}}-\ln |g|\right)=\left(\exp \circ \chi_{\{q\}}\right) /|g| \notin \mathcal{Q},
$$

and consequently, $\left(\exp \circ \chi_{\{q\}}\right) / g \notin \mathcal{Q}$.

Theorem 2.7. $\mathrm{q}(\mathcal{Q})=\omega$.

Proof. The inequality $\mathrm{q}(\mathcal{Q}) \geq \omega$ follows by Theorem 2.4 and Corollary 2.5. The opposite inequality follows by Proposition 2.6.

\section{Darboux quasi-continuous functions}

Now we turn to the quotients of Darboux quasi-continuous functions. Denote by $\mathcal{B}^{\star}$ the family of all cliquish functions $f$ such that

a) both $[f>0]$ and $[f<0]$ are semi-open;

b) if $a<b$ and $f(a) f(b)<0$, then $[f=0] \cap(a, b) \neq \emptyset$;

c) both $[f>0]$ and $[f<0]$ are bilaterally dense in itself.

Proposition 3.1. $\mathcal{D} \mathcal{Q} / \mathcal{D} \mathcal{Q} \subset \mathcal{B}^{\star}$

Proof. Let $f \in \mathcal{D} \mathcal{Q} / \mathcal{D} \mathcal{Q}$. Evidently $f \in \mathcal{D} / \mathcal{D} \cap \mathcal{Q} / \mathcal{Q}$. So by Proposition 2.1 and [13], $f$ is cliquish and conditions b) and c) hold. Choose $g \in \mathcal{Q}$ and $h \in$ $\mathcal{D}$ such that $f=g / h$. We may assume that $h>0$. Then $[f>0]=[g>0]$ and $[f<0]=[g<0]$. Now condition a) follows by [14]. 
Lemma 3.2. Let $f_{1}, \ldots, f_{l} \in \mathcal{C}_{q}$, and assume that each $f_{i}$ is either positive or negative on $(a, b)$. There is a Baire one function $g:(a, b) \rightarrow(0, \infty)$ such that for each $i,\left(\left|f_{i}\right| / g\right)[(a, c)]=\left(\left|f_{i}\right| / g\right)[(c, b)]=(0, \infty)$ for each $c \in(a, b)$, and $f_{i} / g$ is both Darboux and quasi-continuous.

Proof. First we proceed as in the proof of Lemma 2.3 to construct the function $\tilde{g}: \mathbb{R} \rightarrow \mathbb{R}$. Define $g=\exp \circ \tilde{g}\lceil(a, b)$. One can easily see that then the requirements of the lemma are fulfilled.

Theorem 3.3. Let $f_{1}, \ldots, f_{k} \in \mathcal{B}^{\star}$. There is a Baire one function $g: \mathbb{R} \rightarrow$ $(0, \infty)$ such that $f_{i} / g \in \mathcal{D} \mathcal{Q}$ for each $i$.

Proof. Define $U$ by $(1)$, and let $\left\{\left(a_{n}, b_{n}\right): n<r\right\}$ be a sequence of all its components. By Lemma 3.2, for each $n$ there is a function $g_{n}:\left(a_{n}, b_{n}\right) \rightarrow$ $(0, \infty)$ such that for each $i, f_{i} / g_{n}$ is both Darboux and quasi-continuous on $\left(a_{n}, b_{n}\right)$ and if $f_{i}$ is nonzero on $\left(a_{n}, b_{n}\right)$, then

$$
\begin{aligned}
& \left(\left|f_{i}\right| / g_{n}\right)\left[\left(a_{n}, c\right)\right] \\
& =\left(\left|f_{i}\right| / g_{n}\right)\left[\left(c, b_{n}\right)\right]=(0, \infty) \quad \text { for each } c \in\left(a_{n}, b_{n}\right) .
\end{aligned}
$$

Define

$$
g(x)= \begin{cases}g_{n}(x) & \text { if } x \in\left(a_{n}, b_{n}\right), n<r \\ 1 & \text { otherwise }\end{cases}
$$

Fix $i \in\{1, \ldots, k\}$. The proof of quasi-continuity of $f_{i} / g$ is a repetition of the argument used in Theorem 2.4. The only difference is that if $x \in \operatorname{clint}\left[f_{i} \neq 0\right]$ and $f_{i}(x) \neq 0$, then we require that $\operatorname{sgn} \circ f_{i}=\operatorname{sgn} f_{i}(x)$ on $\bigcup_{m \in \mathbb{N}} I_{m}$. (We use conditions (3) and a)).

Finally we will show that $f_{i} / g \in \mathcal{D}$. Fix $a<b$ and let $I=[a, b]$. Clearly $f_{i} / g$ is Darboux on each connected component of $U$, so we may assume that $I \backslash U \neq \emptyset$. Define $A=I \cap\left[f_{i}>0\right]$ and $B=I \cap\left[f_{i}<0\right]$. First we will show that

$$
\text { if } A \neq \emptyset \text {, then }\left(f_{i} / g\right)[I] \supset(0, \infty) \text {. }
$$

Indeed, if $A \neq \emptyset$, then by c), $(a, b) \cap\left[f_{i}>0\right] \neq \emptyset$. Now since $\left[f_{i}>0\right]$ is semi-open and $U$ is dense in $\mathbb{R}$, there is an $n<r$ such that $J \stackrel{\text { df }}{=}(a, b) \cap$ $\operatorname{int}\left[f_{i}>0\right] \cap\left(a_{n}, b_{n}\right) \neq \emptyset$. Observe that $I \cap\left\{a_{n}, b_{n}\right\} \neq \emptyset$ (recall that $I \backslash U \neq \emptyset$ ) and $f_{i} / g=f_{i} / g_{n}$ on $J$. Finally by $(3),\left(f_{i} / g\right)[I] \supset\left(f_{i} / g\right)[J]=(0, \infty)$.

Similarly we can show that if $B \neq \emptyset$, then $\left(f_{i} / g\right)[I] \supset(-\infty, 0)$. Now we consider four cases.

If $A=\emptyset=B$, then $\left(f_{i} / g\right)[I]=\{0\}$.

If $A \neq \emptyset=B$, then $(0, \infty) \subset\left(f_{i} / g\right)[I] \subset[0, \infty)$, so $\left(f_{i} / g\right)[I]$ is an interval. Analogously we proceed if $A=\emptyset \neq B$. 
If $A \neq \emptyset \neq B$, then $\left(f_{i} / g\right)[I] \supset(-\infty, 0) \cup(0, \infty)$. But by b), $0 \in f_{i}[I]$. Consequently, $\left(f_{i} / g\right)[I]=\mathbb{R}$.

The next corollary follows by Proposition 3.1 and Theorem 3.3. Its proof is analogous to that of Corollary 2.5.

Corollary 3.4. $\mathcal{D} \mathcal{Q} / \mathcal{D Q}=\mathcal{B}^{\star}$.

Theorem 3.5. $q(\mathcal{D Q})=\omega$.

Proof. The inequality $\mathrm{q}(\mathcal{D} \mathcal{Q}) \geq \omega$ follows by Theorem 3.3 and Corollary 3.4. The opposite inequality follows by Proposition 2.6.

The next proposition shows that $\mathcal{D} \mathcal{Q} / \mathcal{D} \mathcal{Q}$ is a proper subset of $\mathcal{D} / \mathcal{D} \cap \mathcal{Q} / \mathcal{Q}$.

Proposition 3.6. $\mathcal{D} \mathcal{Q} / \mathcal{D} \mathcal{Q} \neq \mathcal{D} / \mathcal{D} \cap \mathcal{Q} / \mathcal{Q}$.

Proof. Let $C$ be the Cantor ternary set and let $C_{0}$ denote the set of points of bilateral accumulation of $C$. Define

$$
f(x)= \begin{cases}1 & \text { if } x \in C_{0}, \\ 0 & \text { if } x \in C \backslash C_{0}, \\ -1 & \text { if } x \in \mathbb{R} \backslash C .\end{cases}
$$

Then $f \in \mathcal{D} / \mathcal{D} \cap \mathcal{Q} / \mathcal{Q}$ (see [13] and Corollary 2.5). On the other hand, $[f>0]=C_{0}$. So by Proposition $3.1, f \notin \mathcal{D} \mathcal{Q} / \mathcal{D} \mathcal{Q}$.

\section{References}

[1] Biswas, N., On some mappings in topological spaces, Bull. Calcutta Math. Soc. 61 (1969), 127-135.

[2] Borsík, J., Products of simply continuous and quasi-continuous functions, Math. Slovaca 45(4) (1995), 445-452.

[3] Ciesielski, K. and Miller, A. W., Cardinal invariants concerning functions whose sum is almost continuous, Real Anal. Exchange 20(2) (1994-95), 657-672.

[4] Fast, H., Une remarque sur la propriété de Weierstrass, Colloq. Math. 7 (1959), $75-77$.

[5] Grande, Z., Sur la quasi-continuité et la quasi-continuité approximative, Fund. Math. 129 (1988), 167-172.

[6] Grande, Z. and Natkaniec, T., Lattices generated by $\mathcal{T}$-quasi-continuous functions, Bull. Polish Acad. Sci. Math. 34 (1986), 525-530.

[7] Kempisty, S., Sur les fonctions quasicontinues, Fund. Math. 19 (1932), 184-197. 
[8] Levine, N., Semi-open sets and semi-continuity in topological spaces, Amer. Math. Monthly 70 (1963), 36-41.

[9] Maliszewski, A., On the limits of strong Światkowski functions, Zeszyty Nauk. Politech. Łódz. Mat. 27(719) (1995), 87-93.

[10] Maliszewski, A., On theorems of Pu \&S Pu and Grande, Math. Bohem. 121(1) (1996), $232-236$.

[11] Maliszewski, A., Darboux property and quasi-continuity. A uniform approach, WSP, Słupsk, 1996.

[12] Natkaniec, T., Products of Darboux functions, Real Anal. Exchange 18(1) (1992-93), $232-236$.

[13] Natkaniec, T. and Orwat, W., Variations on products and quotients of Darboux functions, Real Anal. Exchange 15(1) (1989-90), 193-202.

[14] Neubrunnová, A., On certain generalizations of the notion of continuity, Matemat. Casopis SAV 23(4) (1973), 374-380.

[15] Thielman, H. P., Types of functions, Amer. Math. Monthly 60(3) (1953), 156-161.

JOLANTA JAŁOCHA

DEPARTMENT OF MATHEMATICS

PL. WEYSSENHOFFA 11

85-072 BydGOSZCZ

POLAND 